\title{
Solar system astronomy with the 3.6-m DOT and the 4-m ILMT
}

\author{
Shashikiran Ganesh ${ }^{1 *}$, Kumar Venkataramani ${ }^{1,2}$, Kiran Singh Baliyan ${ }^{1}$, \\ Umesh Chandra Joshi ${ }^{1}$
}

${ }^{1}$ Physical Research Laboratory, Navarangpura, Ahmedabad, India

${ }^{2}$ Indian Institute of Technology, Gandhinagar

\begin{abstract}
Solar system astronomy would be an important field of study with the 3.6-m Devasthal Optical Telescope (DOT) and the 4-m International Liquid Mirror Telescope (ILMT). In this contribution, we highlight the work that could be done in reaching a better understanding of the Solar system and its constituents - particularly the minor bodies and other smaller objects. There may be a large number of very faint objects in the vicinity of the Earth orbit. In fact only recently a 'second moon' of the Earth has been found and has been designated 2016 HO3. This is a quasi-satellite with the same period of revolution around Earth and Sun. There could be many such objects and it is important to have a full characterization and understanding of these potentially hazardous objects. They are generally fainter than 18th magnitude and one would need a lot of telescope time to fully characterize these objects using techniques of spectropolarimetry. In a similar fashion, a deep census of the Kuiper Belt Objects and the TNOs is needed. In this census, the concept of pencil beam surveys could be extended to cylindrical transit imaging technique available with the 4-m ILMT.
\end{abstract}

\section{Introduction}

One studies the members of the Solar system to understand how the Solar system formed and evolved over time. Thus a study of the least weathered objects, e.g. comets from the Oort cloud which have remained far from the Sun for the longest period of time, would be a study of the primordial material from which the Solar system formed. The bulk of the comet studies are done with small 1-m class telescopes. Polarimetry of comets has been extensively carried out by Joshi and collaborators (e.g. Joshi et al. 2010) and other workers. Recently, Venkataramani et al. (2016) studied comet C/2014 Q2 (Lovejoy) spectroscopically with a 50-cm telescope. However, such studies are quite photon hungry and demand a lot of observing time or larger aperture telescopes. Indeed, with the 1-m class telescopes only the very bright objects could be studied. This means that by the time their study is possible, they are already quite close to the Sun and have evolved quite a bit and developed a coma and tail. In order to study the comets before they start becoming active one has to turn to larger telescopes for their larger capacity to collect light.

In the present contribution, we briefly discuss results from polarimetric observations of comets. We also present a few results from our own spectroscopic observations of comets and asteroids with a

*email: shashi@prl.res.in 


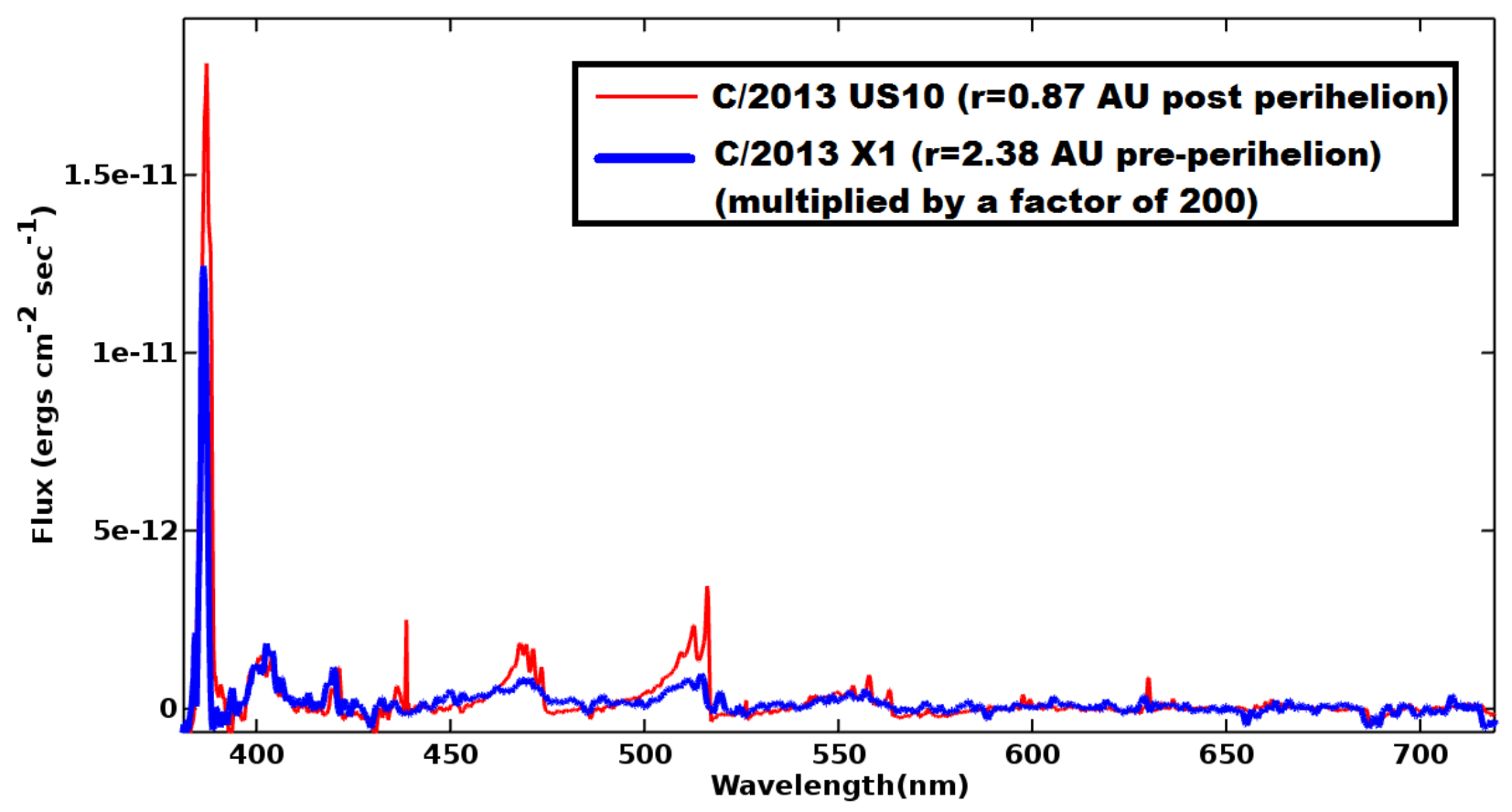

Figure 1: Optical spectra for comets C/2013 US10 (Catalina) and C/2013 X1 (PanSTARRS) observed on 30 November 2015 with the LISA spectrograph mounted on the 50-cm telescope of PRL.

50-cm telescope. With the backdrop of these results, we discuss how spectropolarimetry of the minor bodies of the Solar system would be an important aspect for Solar system studies with the 3.6-m DOT of the Aryabhatta Research Institute of Observational Sciences (ARIES).

The Oort cloud is a spherical cloud surrounding the Sun, hypothesized to be the source of the long period comets. It is about a thousand times more distant than the region occupied by the Kuiper Belt Objects (KBOs), the Trans Neptunian Objects (TNOs) and objects of the scattered disk. One discovers the members of these regions only when they are perturbed and sent into the inner Solar system as long period comets or comets with hyperbolic orbits. The concept of 'pencil beam surveys' has been used in the past to statistically study the fainter members of the Kuiper Belt (e.g. Luu \& Jewitt, 1998). The repeated (at daily interval) transit imaging capability of the 4-m ILMT would highly enhance our understanding of the fainter population of KBOs, TNOs etc It is expected that a large number of new objects would be detected from the survey images. An automated technique to detect and announce possible new objects would be very useful to allow timely follow up of these sources to determine their orbital parameters.

\section{The case for spectropolarimetry of Solar system minor bodies with the 3.6-m DOT}

\subsection{Spectroscopy of comets and asteroids}

In Fig. 1, we show two spectra for comets C/2013 US10 (Catalina) and C/2013 X1 (PanSTARRS) with PRL's 50-cm telescope and the LISA spectrograph ${ }^{1}$ operated at the Mount Abu Infrared Observatory (MIRO). The spectra were taken on 30 November 2015 when the comets were 0.87 and 2.38 AU

\footnotetext{
${ }^{1}$ LISA is a low resolution spectrograph manufactured by M/s Shelyak Instruments http : / / www . shelyak. com/
} 


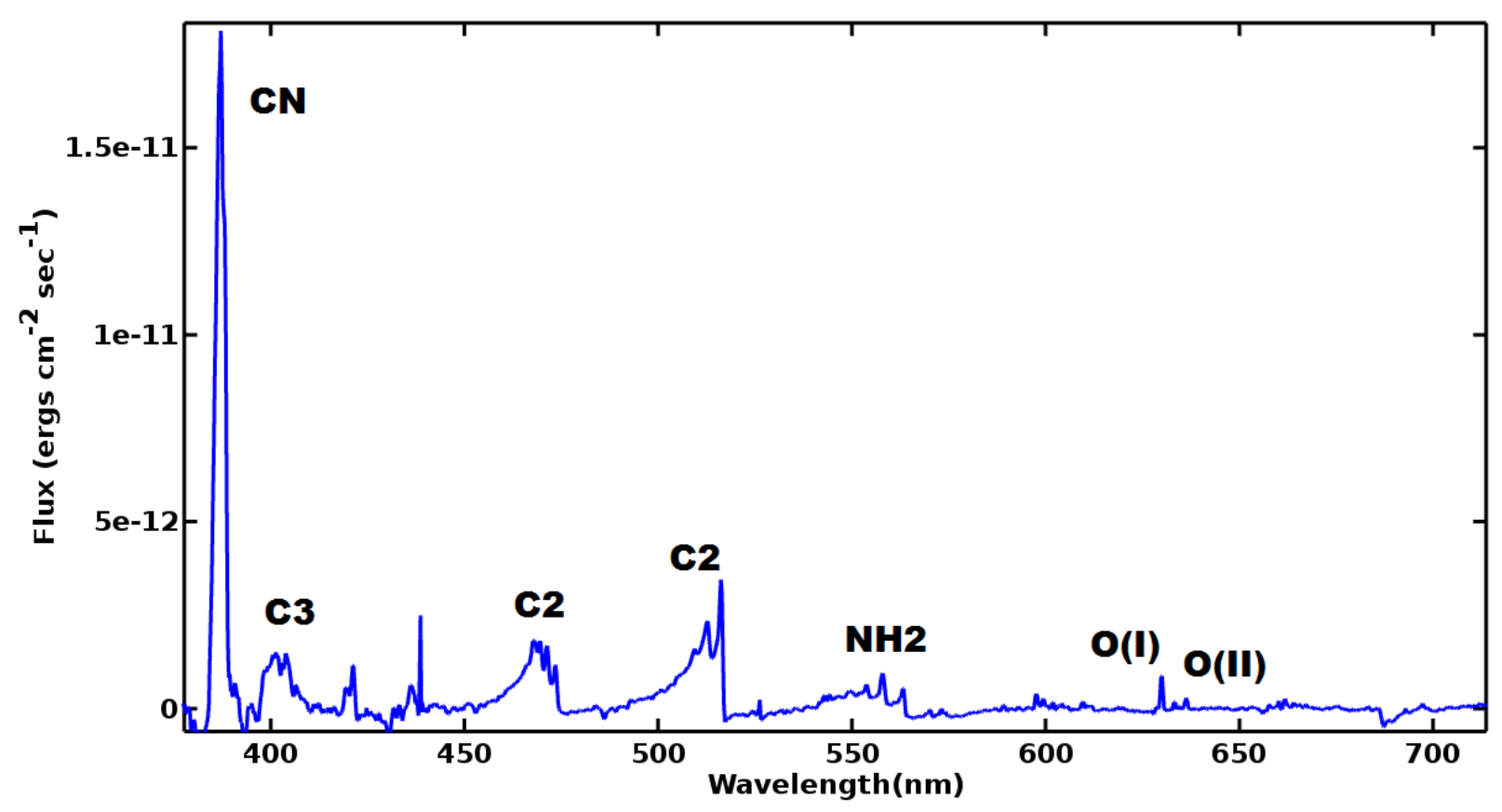

Figure 2: Molecular bands as seen in the spectrum of comet C/2013 US10 (Catalina).

after and before perihelion respectively. In this figure, the two spectra are shown with the continuum spectrum subtracted. $\mathrm{C} / 2013 \mathrm{X} 1$ is much fainter (being more distant and inbound towards perihelion) and its spectrum is multiplied by a factor of 200 in Fig. 1. Comet C/2013 X1 was at magnitude 11.57 and C/2013 US10 was much brighter at 7.9. A large number of molecular bands are seen in the spectra (as in Fig. 2). Venkataramani et al. (2016) studied the evolution of the molecular emissions at several epochs in the case of bright comet C/2014 Q2 (Lovejoy). They determined the production rates as well as the scale lengths of these molecules and studied how the production rates changed with time. The comet was found to be more active after perihelion. One of the constraints with a small telescope is that such studies can be carried out only when the comets have become bright (and hence also become active) enough for spectroscopy.

In Fig. 3 we show the spectra of asteroid 2015 TB145 taken with PRL's 50-cm telescope and the LISA spectrograph during the close flyby on 30 October 2015. Two spectra with an exposure time of 30 minutes were co-added for this asteroid. We also overplot the spectrum of asteroid Vesta which was taken with a 50 second exposure on 29 October 2015. These spectra are sky subtracted. Asteroid 2015 TB145 was discovered on 10 October 2015. It is an Apollo near-Earth asteroid approximately 600 meters in diameter. At closest approach (31 October 2015 at 17:00 UT) it was at a distance of 1.27 lunar diameter. Earlier there was some ambiguity about its identity - comet or asteroid? - due to its large inclination and eccentricity. The spectrum is clearly that of an asteroid as can be seen from the complete absence of molecular emission. The asteroid was close to magnitude 11 at the time of these observations while Vesta was nearly of 7 th magnitude.

Characterizing such objects, which are generally faint (absolute magnitude, $\mathrm{H}$, of 20) requires the resources of a large telescope. The late discovery (just days before flyby) shows that there could be many such objects awaiting discovery. Continued follow-up observations for this asteroid and other similar objects is important to settle the question whether they are intrinsically rocky asteroids or 'dead' comets with very little activity left after many close perihelion passes. One example of a 'dead' comet which was found to become 'active' again is comet 49P/Arend-Rigaux. Images from the MIRO 1.2-m telescope showed this object to exhibit a jet without a coma/tail during its last perihelion 


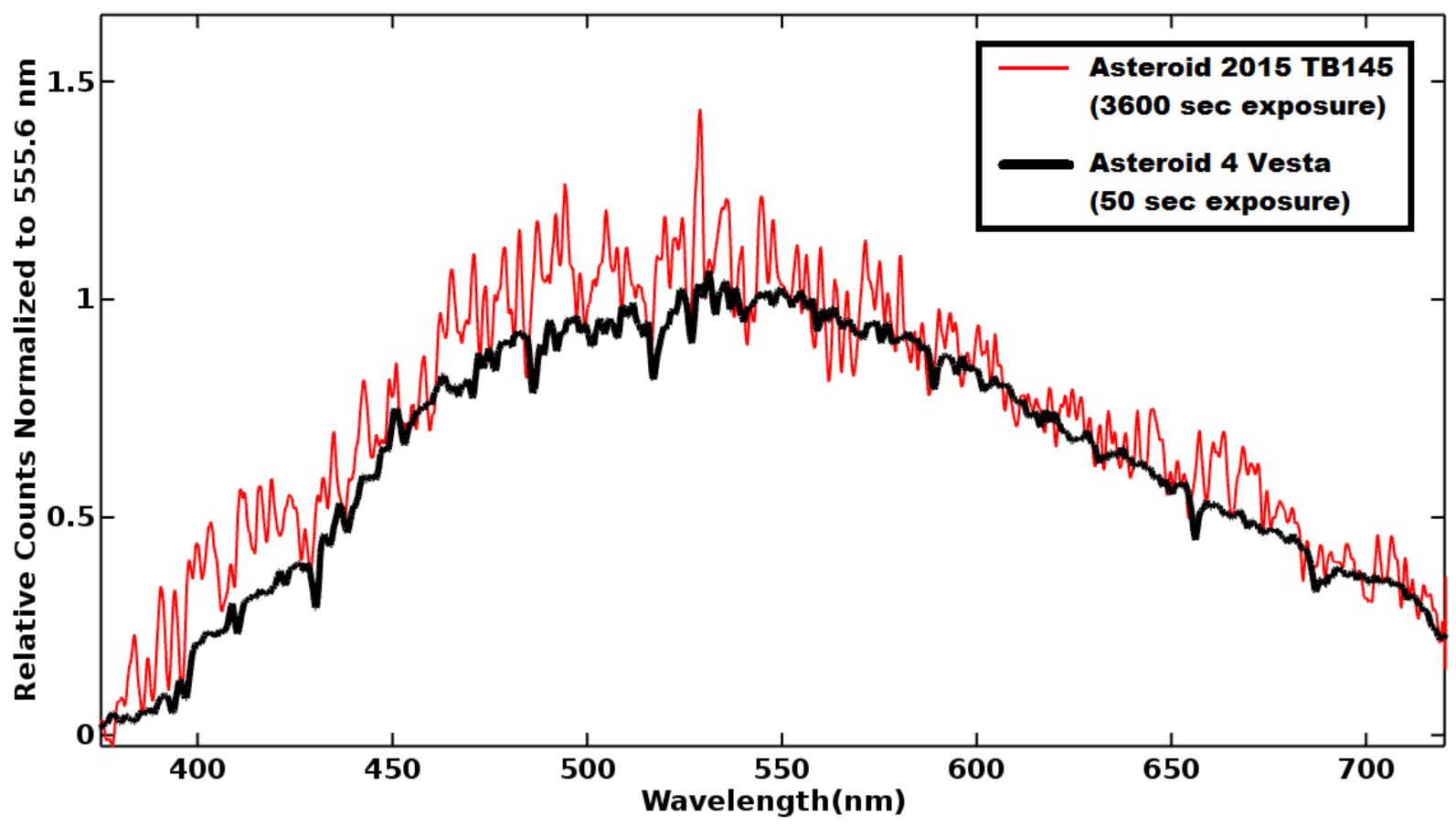

Figure 3: Optical spectrum for the asteroids 2015 TB145 (red line) and Vesta (black line) as observed with the LISA spectrograph mounted on the 50-cm PRL telescope on 30 October 2015 and 29 October 2015, respectively.

pass. On a previous pass (2006) it had been studied by the Spitzer Space Observatory. Combining these data with laboratory results, Sivaraman et al. (2015), showed the detection of amorphous water ice on the surface of this comet. This indicates that even 'dead' comets are worth monitoring.

\subsection{Polarimetric results}

Cometary polarimetry has been used extensively to study the properties of the dust constituting the cometary coma. Kiselev et al. (2005) have compiled all the polarimetric observations of comets (2600 observations over 65 years for 64 different comets). In Fig. 4 we show the polarization phase curve for comets studied using red filters such as the $684 \mathrm{~nm}$ filter defined by the International Halley Watch/International Astronomical Union (IHW/IAU). The observations for comet Hale-Bopp (filled diamond symbols) show a significantly higher polarization than all the other comets (open circles). A large coverage in phase angle and wavelength (at many continuum points) is needed to understand the dust characteristics in the coma of comets.

The IHW filter set consists of several narrow band 'continuum' filters and also filters covering the prominent molecular emissions. The drawbacks in these filters were addressed and a new set of filters were prepared during the very well observed apparition of the comet Hale-Bopp. However, both of these filter sets are now quite old and their transmission properties have probably changed compared to the design specifications.

\subsection{Long slit spectropolarimetry at low to medium resolution}

From the previous subsections it is clear that there is an urgent need to study a good sample of objects (comets/asteroids) with a reasonably large telescope with long slit spectroscopy for the study 


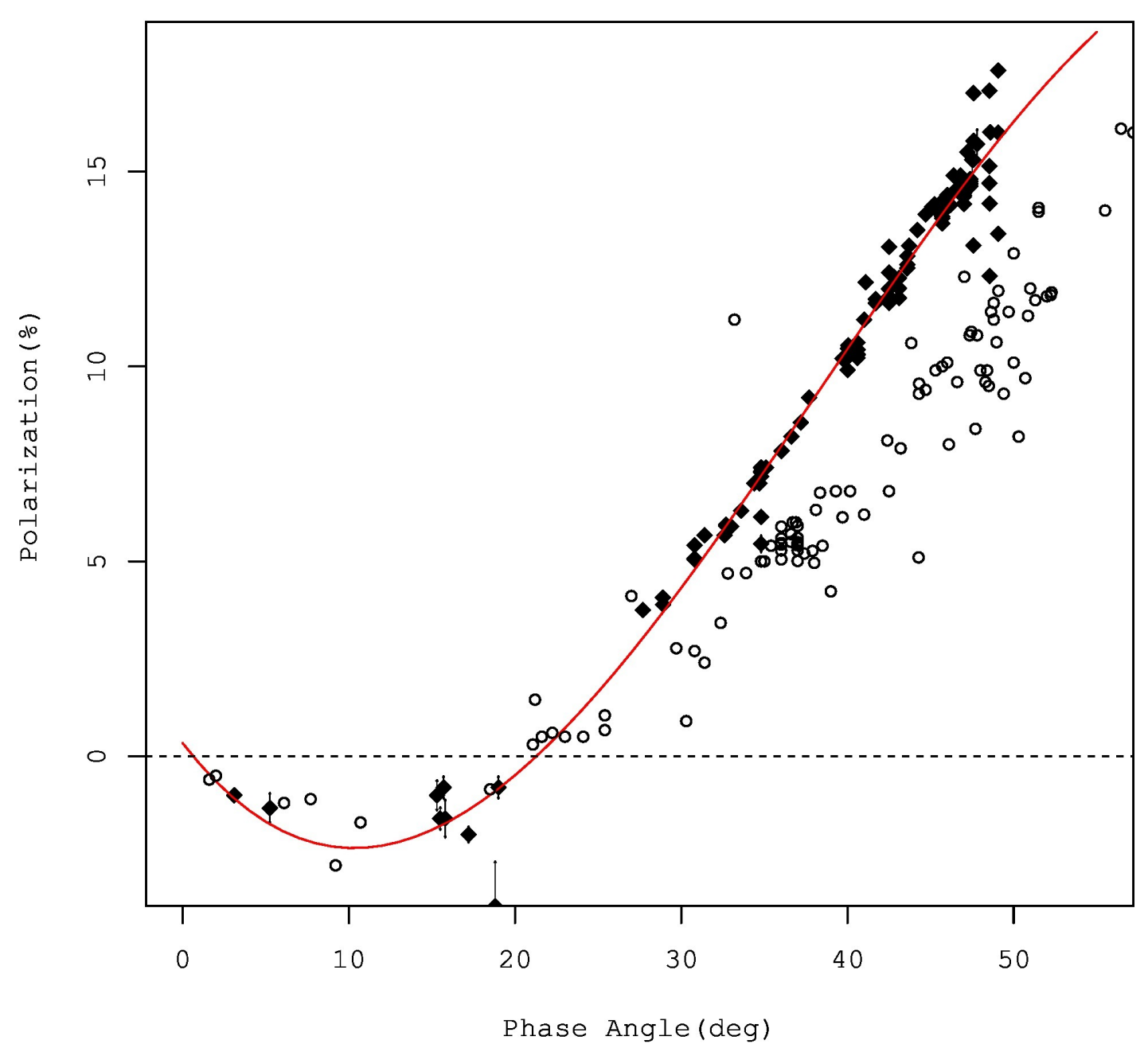

Figure 4: Polarization phase curve in the $684 \mathrm{~nm}$ International Halley Watch (IHW) filter. The filled diamonds are for comet Hale-Bopp while the open circles are for other comets. See text for discussion and references.

of extended objects. Comets need to be studied at large distances (more than $3 \mathrm{AU}$ ) when they are in bound towards perihelion. Large telescopes would enable such studies efficiently. Ideally such spectroscopic observations need to be combined with polarimetry through spectropolarimetric instruments. With such instruments one could study the molecular and dust evolution of the cometary comae at large distances from the Sun. Spectropolarimetry would also provide an unambiguous method to measure polarization at continuum points. Well designed instruments could have photon noise limited performance on the 3.6-m DOT.

With high resolution cometary spectra becoming available, a new narrow band filter set would need to be defined for studying the spatial distribution and evolution of the molecules ejected from the cometary nucleus. Typically these filters have a band width of 4 to $8 \mathrm{~nm}$. Similarly, the continuum filters would be useful to study the dust characteristics and their evolution with distance from the nucleus. Both of these narrow band imaging projects need the light gathering power of the kind available with the 3.6-m DOT. For efficient usage of telescope time, it would be very useful to have a 
wide-field multi-band imager using multiple CCDs and suitable beam-splitter + filter combination.

Spectroscopy, spectropolarimetry as well as narrow band imaging techniques are complementary and all demand large apertures and efficient instruments so as to cover a large range of minor bodies especially at the less studied fainter end of the distribution.

\section{Surveying for minor bodies with the 3.6-m DOT and the 4-m ILMT}

At the high mass end, the census of Solar system bodies is fairly complete, although there are recent articles hinting at the possibility of a ninth planet of similar mass as the other gas giants. At the lower mass extreme, the situation is quite different and surveys such as the PanSTARRS and the Catalina Sky Survey continue to find objects including Near Earth Objects at regular intervals.

\subsection{Pencil beam surveys}

During the late 1990s, 'pencil beam surveys' were carried out (e.g. Chiang \& Brown 1999) using the Keck 10-m telescope to look for faint Kuiper Belt Objects with a visual magnitude $m_{V}$ between 25 and 30. Two objects were discovered in that specific work. Recent results can be found in Parker \& Kavelaars (2010), who look to provide limits on the distant populations of the Trans Neptunian Objects. One of the newest references on this line of work is that of Vaduvescu et al. (2017) who used images taken by the Subaru telescope with its Suprime-Cam. After accounting for already known asteroids in the 2018 moving objects measured over an area of 16.6 square degree, they were able to identify 18 Near Earth Asteroid (NEA) candidates. They conclude that 'using the $R_{c}$ filter in good weather conditions, mostly dark time and sky directions slightly biased towards the ecliptic, at least one NEA could be discovered in every 1 square degree surveyed.' Note that this conclusion only holds for a 8-m telescope.

\subsection{Continuous imaging - detecting distant minor bodies with their movement across the field of view}

The 4-m ILMT coming up at Devasthal (India) is a transit imaging telescope with a fixed field of view with a width of nearly half a degree. The exposure time is limited by the time an object takes to drift across the 4096 pixels of the CCD. However, this exposure time can be increased by co-adding data from successive nights.

With transit imaging of a narrow strip with declination equal to the local latitude, the telescope's field of view would move twice across the galactic plane in an year. Similarly, the ecliptic region, too, would transit (at midnight) across the telescope during the month of December and hence this part of the ecliptic would be observable for several months during clear weather. The single 'frame' of the 4-m ILMT image is expected to reach a limiting magnitude of $m_{I}^{\prime}=22$. It should, therefore, be capable to detect a good sample of Solar system bodies within the narrow declination strip available to the 4-m ILMT. The more distant ones are more likely to be detected with their relatively slow motion, across the field of view, with time. An algorithm would need to be put in place to automatically detect such moving objects so that they may be followed up (with the 3.6-m DOT if needed) before they move out of the declination strip observable with the 4-m ILMT. This would help provide a better understanding of the completeness of information in the corresponding magnitude range of the Solar system bodies (NEAs, Comets, KBOs, TNOs, etc). 


\section{Conclusion}

In this contribution we provide a flavour of the types of studies of the minor bodies of the Solar system where the new 3.6-m DOT and 4-m ILMT could provide substantial contributions to our understanding.

\section{Acknowledgements}

We thank the organizers of the 1st BINA workshop for supporting our stay at Nainital and for very useful discussions. Work at PRL is supported by the Dept. of Space, Govt. of India.

\section{References}

Chiang E. I., Brown M. E. 1999, AJ, 118, 1411

Joshi U .C., Ganesh S., Baliyan K. S. 2011, MNRAS, 412, L58

Luu J. X., Jewitt D. C. 1998, ApJ, 502, L91

Parker A. H., Kavelaars J. J. 2010, Icarus, 209, 766

Sivaraman B., Venkataraman V., Kalyaan A., Arora S., Ganesh S. 2015, AdSpR, 56, 2428

Vaduvescu O., Conovici M., Popescu M. et al. 2017, AN, 338, 527

Venkataramani K., Ghetiya S., Ganesh S. et al. 2016, MNRAS, 463, 2137 